\title{
Observation of coherent Cerenkov radiation from a solid dielectric with short bunches of electrons
}

\section{$\operatorname{AUTHOR}(\mathrm{S}):$}

Takahashi, T; Shibata, Y; Ishi, K; Ikezawa, M; Oyamada, M; Kondo, $\mathrm{Y}$

\section{CITATION:}

Takahashi, T ... [et al]. Observation of coherent Cerenkov radiation from a solid dielectric with short bunches of electrons. Physical Review E 2000, 62(6): 8606-8611

\section{ISSUE DATE:}

2000-12

URL:

http://hdl.handle.net/2433/39900

RIGHT:

Copyright 2000 American Physical Society 


\title{
Observation of coherent Čerenkov radiation from a solid dielectric with short bunches of electrons
}

\author{
Toshiharu Takahashi \\ Research Reactor Institute, Kyoto University, Kumatori, Osaka 590-0494, Japan \\ Yukio Shibata, Kimihiro Ishi, and Mikihiko Ikezawa \\ Research Institute for Scientific Measurements, Tohoku University, Katahira, Aoba-ku, Sendai 980-8577, Japan \\ Masayuki Oyamada \\ Laboratory of Nuclear Science, Tohoku University, Mikamine, Taihaku-ku, Sendai 982-0826, Japan \\ Yasuhiro Kondo \\ Faculty of Engineering, Tohoku University, Aramaki, Aoba-ku, Sendai 980-8579, Japan
}

(Received 12 July 2000)

\begin{abstract}
Short bunches of $150-\mathrm{MeV}$ electrons of a linear accelerator passed along the surface of a crystal quartz or a teflon and coherent Čerenkov radiation from the solid dielectrics has been observed in the wavelength range from 0.5 to $4 \mathrm{~mm}$. Properties of the radiation have been experimentally investigated. The angular distribution of the observed radiation showed a maximum peak in the direction of the Cerenkov angle with several satellite peaks. The intensity increased linearly with increasing the length of the medium and was proportional to the square of the number of electrons in the bunch. The spectral intensity was enhanced by almost five orders of magnitude in comparison with the theoretical calculation of incoherent radiation.
\end{abstract}

PACS number(s): 41.60.Bq, 07.57.Hm, 41.75.Ht, 52.75.Va

\section{INTRODUCTION}

In recent years there has been a growing interest in coherent radiation from short bunches of electrons as the intense source of millimeter and submillimeter waves and as the high-resolution monitor of the bunch form. Several types of coherent radiation have been experimentally investigated so far, such as synchrotron radiation [1-5], transition radiation [6-10], diffraction radiation [11], and Smith-Purcell radiation $[12,13]$. Coherent transition radiation has been recently used as a light source for a material science in the millimeter-wave region [14], and the prebunched free electron laser using coherent synchrotron radiation has been experimentally investigated $[15,16]$.

Another radiation from a high-energy electron beam is Cerenkov radiation. In a dielectric medium, Cerenkov radiation is emitted [17] when the velocity of an electron $v$ exceeds that of light $c / n$, that is,

$$
\beta n>1,
$$

where $n$ is the refractive index of the medium and $\beta=v / c$. The condition that $\beta n=1$ is called the Cerenkov threshold which is defined in a medium with an infinite extent, and the condition $\beta n>1$ is called the Cerenkov criterion.

Some research groups were tried to observed coherent Cerenkov radiation from a finite trajectory of electrons in a gas in the microwave and the millimeter-wave region $[6,8,18-20]$. Through these experiments a problem about the nomenclature of the radiation in a gas has been emerged. The observed radiation was called Cerenkov radiation $[18-20]$ according to conventional nomenclature with the Cerenkov criterion on the one hand, and also called transition radiation $[6,8]$ from the property of observed radiation on the other hand.

According to our recent study on Čerenkov radiation in gas [21], the confusion of the nomenclature has been attributed to the lack of the path length $(L)$ in gas. The Cerenkov criterion $\beta n>1$ have generally been taken to be the only condition but the criterion has been insufficient to a medium with a finite length, especially when the refractive index is close to 1 . On the basis of the consideration of the formation zone $L_{f}=\beta \lambda /|1-\beta n \cos \theta|$, the additional criterion $L>L_{f}$ for the Cerenkov radiation has been proposed in our previous paper [21], where $\theta$ is the angle between a direction of observation and the electron trajectory. Since the refractive index of gas is close to 1 the value of $L_{f}$ of air for $150-\mathrm{MeV}$ electrons, for example, exceeds $10 \mathrm{~m}$ in the millimeter-wave region. Under this alternate criterion the radiation called Cerenkov radiation in some papers [18-20] should be interpreted as transition radiation. To observe Cerenkov radiation in the long-wavelength region, a solid dielectric with a large refractive index need to be used to get a small value of $L_{f}$.

In the case of forward observation $(\theta \approx 0)$, the strictest criterion within $L>L_{f}$ is given by

$$
\beta n>1+\frac{\beta \lambda}{L}
$$

This criterion can be easily satisfied for solid dielectrics with the refractive index larger than unity. In the case of gas, on the other hand, $n$ is close to unity and the second term on Eq. (1.2), $\beta \lambda / L$, poses a severe restriction. 
Using a solid dielectric, the enhancement of the intensity of Cerenkov radiation from bunched electrons of $1 \mathrm{MeV}$ or less was observed hitherto [22,23], but no detailed study was made. The aim of our experiment in this paper is to clarify properties of coherent Čerenkov radiation from short bunches of high-energy electrons of a linear accelerator.

\section{THEORY OF COHERENT ČERENKOV RADIATION}

\section{A. Čerenkov radiation in a dielectric medium}

According to Tamm's theory [24], the intensity of Črenkov radiation from a finite trajectory $(L)$ of an electron is given by [25]

$$
\begin{aligned}
& \frac{d^{2} P_{0}}{d \Omega d \lambda}=\frac{\alpha n}{\lambda}\left(\frac{L}{\lambda}\right)^{2}\left(\frac{\sin X(\lambda, \theta)}{X(\lambda, \theta)}\right)^{2} \sin ^{2} \theta, \\
& X(\lambda, \theta)=\frac{\pi L}{\beta \lambda}(1-\beta n \cos \theta),
\end{aligned}
$$

where $\alpha$ is the fine-structure constant, $\lambda$ a wavelength in vacuum, $\beta$ the ratio of the velocity of the electron to that of light, and $\theta$ the angle between a direction of observation and the electron trajectory. The intensity is represented by the number of photons $\left(P_{0}\right)$ per unit wavelength $(d \lambda)$ and unit solid angle $(d \Omega)$. The observation point is assumed to be far from the radiation source. Equation (2.1) shows that the radiation intensity is not extinguished and remains finite even if $\beta n<1$. When $|\beta n-1| L / \lambda \gg 1$, Eq. (2.1) is integrated over all solid angles in two cases. For $\beta n>1$,

$$
\frac{d P_{0}}{d \lambda}=\frac{2 \pi \alpha L}{\lambda^{2}}\left(1-\frac{1}{\beta^{2} n^{2}}\right)+\frac{2 \alpha}{\pi n \lambda}\left(\frac{1}{\beta n} \ln \frac{1+\beta n}{|1-\beta n|}-2\right)
$$

and for $\beta n<1$

$$
\frac{d P_{0}}{d \lambda}=\frac{2 \alpha}{\pi n \lambda}\left(\frac{1}{\beta n} \ln \frac{1+\beta n}{1-\beta n}-2\right) .
$$

The first term of Eq. (2.3) represents the intensity of "pure" Cerenkov radiation from a continuous medium and it is proportional to L. Equation (2.4) and the second term of Eq. (2.3) result from the ends of the trajectory. In Tamm's theory the electron is assumed to have a constant velocity $\beta c$ only during traveling the length $L$. Since this condition corresponds to the trajectory limited by perfect conductors on both sides, origin of the radiation given by Eq. (2.4) and the second term of Eq. (2.3) should be due to transition radiation from a metallic boundary.

On the other hand, when $|\beta n-1| L / \lambda \gg 1$ is not fulfilled, integration of Eq. (2.1) cannot be distinctly divided in two terms of Čerenkov and transition radiation. For $n \simeq 1, \beta$ $\simeq 1$, and a small angle $\theta$, i.e., $|\beta n-1| L / \lambda \ll 1$, Eq. (2.1) is approximately agreement with that of transition radiation [21].

\section{B. Čerenkov radiation from a nearby solid dielectric}

When the electron beam is passed near a surface outside a dielectric slab, the intensity of Cerenkov radiation, Eq. (2.1), is multiplied by a coupling factor [26]

$$
K=\exp \left(-4 \pi \frac{a}{\gamma \beta \lambda}\right),
$$

where $a$ is the distance between the electron beam and the surface, and $\gamma$ is the Lorentz factor $\gamma=\left(1-\beta^{2}\right)^{-1 / 2}$.

With a sylindrical tube the coupling factor is expressed as

$$
K=\int g(\rho) \exp \left(-4 \pi \frac{R-\rho}{\gamma \beta \lambda}\right) \rho d \rho,
$$

where $g(\rho)$ is the transverse distribution of the electron beam and $R$ is the radius of the cylindrical hole. When the transverse distribution is uniform within a circle of radius $D$ and $D \geqslant R$, the factor $K$ is approximated as

$$
K=2\left(\frac{\gamma \beta \lambda}{4 \pi D}\right)^{2}\left\{\exp \left(-\frac{4 \pi R}{\gamma \beta \lambda}\right)+\frac{4 \pi R}{\gamma \beta \lambda}-1\right\}+\left(1-\frac{R^{2}}{D^{2}}\right) .
$$

Since the value of $K$ are larger than 0.9 for the $150-\mathrm{MeV}$ electron beam in the millimeter-wave region, the decrease of the radiation power is negligible even if an electron beam does not pass inside a medium.

\section{Coherent radiation from a small bunch}

The intensity of coherent radiation generated by a short bunch of electrons is given by [27]

$$
P=\left|\sum_{j=1}^{N} \mathbf{E}_{j} \exp \left(i 2 \pi \frac{\mathbf{n} \cdot \mathbf{x}_{j}}{\lambda}\right)\right|^{2}
$$

where $N$ is the number of electrons in a bunch, $\mathbf{E}_{j}$ the electric vector of radiation induced by the $j$ th electron, $\mathbf{x}_{j}$ the position vector of the $j$ th electron, and $\mathbf{n}$ the unit vector along the direction of observation.

The degree of the coherence effect depends not only on the longitudinal size of the bunch but also on the emittance, or the transverse size and the angular divergence, of the electron beam $[9,27]$. We assume that the distribution of electrons in a bunch has cylindrical symmetry. Then, Eq. (2.8) is calculated as $[27,28]$

$$
P=N\left(1+N f_{L} f_{T} \chi\right) P_{0},
$$

where $f_{L}$ is a longitudinal bunch form factor, $f_{T}$ a transverse one, and $\chi$ a factor of an electron-beam divergence. The number $N$ is assumed to be much larger than unity. The function $P_{0}$ is the intensity of Cerenkov radiation emitted by a single electron and corresponds to $P_{0}$ of Eq. (2.1). The values of the factors $f_{L}, f_{T}$, and $\chi$ vary from zero (incoherence limit) to unity (coherence limit). With the minimum and maximum values of these factors, Eq. (2.9) is reduced to

$$
P= \begin{cases}N P_{0} & \text { (incoherence limit) } \\ N^{2} P_{0} & \text { (coherence limit) }\end{cases}
$$




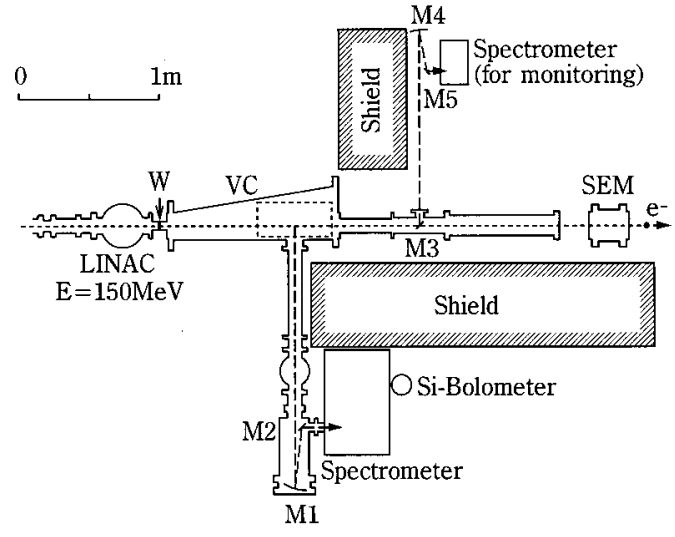

FIG. 1. The arrangement of the experiment. (VC) a vacuum chamber; (W) a titanium window $15 \mu \mathrm{m}$ thick; (M2, M3, M5) plane mirrors; (M4) a spherical mirror; (SEM) a secondary emission monitor; and $\left(e^{-}\right)$electron beam.

In circular cylindrical coordinates $(\rho, \phi, z)$ where the $z$ axis is along the trajectory of the electron beam, the form factors are expressed as [27]

$$
\begin{aligned}
& f_{L}=\left|\int h(z) \exp \left(\frac{i 2 \pi z \cos \theta}{\lambda}\right) d z\right|^{2}, \\
& f_{T}=\left|\iint g(\rho) \exp \left(\frac{i 2 \pi \rho \sin \theta \cos \phi}{\lambda}\right) \rho d \rho d \phi\right|^{2},
\end{aligned}
$$

where $h(z)$ and $g(\rho)$ are the longitudinal and transverse density distribution functions of electrons in the bunch.

The divergence factor is derived from synthesis of vectors as

$$
\chi=\left|\int \mathbf{e} G(\mathbf{u}) d \mathbf{u}\right|^{2}
$$

where $\mathbf{e}$ is the unit vector of an electric field of radiation, $\mathbf{u}$ the unit vector of the direction of motion of an electron, and $G(\mathbf{u})$ the density distribution function of $\mathbf{u}$ in a bunch. In polar coordinates, Eq. (2.13) is written as

$$
\chi=\left\{\iint \frac{\sin \theta \cos \xi-\cos \theta \sin \xi \cos \phi}{\sin \tau} G(\xi) \sin \xi d \xi d \phi\right\}^{2},
$$

TABLE I. The experimental conditions of the electron beam.

\begin{tabular}{lc}
\hline \hline Electron energy $(\mathrm{MeV})$ & 150 \\
Energy spread $(\%)$ & 0.5 \\
Accelerating rf $(\mathrm{GHz})$ & 2.856 \\
Duration of a burst $(\mu \mathrm{s})$ & 2 \\
Repetition rate (pulses/s) & 150 \\
Average beam current $(\mu \mathrm{A})$ & 1 \\
Number of electrons per bunch & $7.2 \times 10^{6}$ \\
Transverse size $2 \rho_{0}(\mathrm{~mm})$ & 7.0 \\
Angular divergence $2 \Psi \quad(\mathrm{mrad})$ & 4.6 \\
Longitudinal bunch length $2 \sigma_{0}(\mathrm{~mm})$ & 0.21 \\
\hline \hline
\end{tabular}

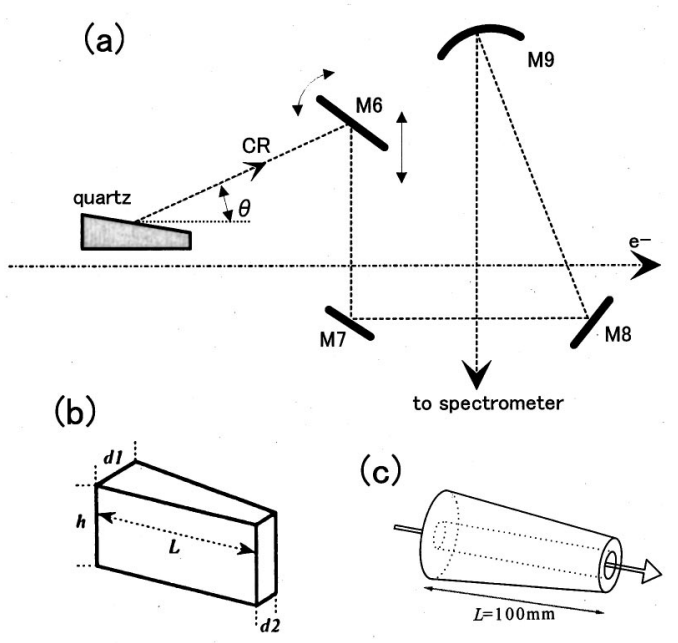

FIG. 2. The schematic view in the vacuum chamber. (a) The sectional diagram of the optical components, (b) the block of quartz, and (c) the cone of teflon with the cylindrical hole of $7 \mathrm{~mm}$. (M6, M7, M8) plane mirrors; (M9) a spherical mirror; and $\left(e^{-}\right)$ electron beam. The values of dimensions in (c) are listed in Table II.

where $\xi$ is the polar angle of $\mathbf{u}$ and $\tau$ is the angle between the direction of motion $\mathbf{u}$ and the direction of observation $\mathbf{n}$.

\section{EXPERIMENTAL PROCEDURES}

The arrangement of the experiment is shown in Fig. 1. The vacuum chamber (VC) was separated from the linac by a titanium window $50 \mu \mathrm{m}$ thick and the pressure in the chamber was kept below $1 \mathrm{~Pa}$. The radiation was reflected by a spherical mirror (M1) which acceptance angle was 70 $\mathrm{mrad}$, led to a grating-type far-infrared spectrometer, and then detected with a liquid-helium-cooled silicon bolometer. The fluctuation of the radiation power was corrected by monitoring the intensity of the coherent transition radiation from the flat aluminum foil $15 \mu \mathrm{m}$ thick (M3). The absolute sensitivity of the measuring system was calibrated by blackbody radiation emitted from a graphite cavity at a temperature of $1200 \mathrm{~K}$ [3]. The secondary emission monitor (SEM) was used for measurement of the electron beam from the linac. The experimental conditions of the Tohoku Linac at Tohoku University are summarized in Table I.

The details in the vacuum chamber is shown in Fig. 2(a). In order to prevent the scattering of the electron beam by a solid dielectric, the electron beam was passed near a surface outside a solid medium for generation of Cerenkov radiation. The electrons move at a distance of $5 \mathrm{~mm}$ from the surface of the quartz [Fig. 2(b)] or move through the cylindrical hole of $7 \mathrm{~mm}$ in diameter of the teflon [Fig. 2(c)]. Figure 2(a) shows the sectional diagram for the quartz. The radiation emitted in the quartz was refracted on the tapered surface, where the angle of the taper was $5.7^{\circ}$ for the quartz. Three kinds of quartz were prepared and these dimensions are listed in Table II. The length of the teflon tube was $100 \mathrm{~mm}$ and the outer diameter was tapered from 30 to $22 \mathrm{~mm}$. The angle of the taper for the teflon was $2.5^{\circ}$. The refractive indices of teflon and quartz for the millimeter wave are 1.4 and 2.1, 
TABLE II. Dimensions of the quartz.

\begin{tabular}{cccc}
\hline \hline$L(\mathrm{~mm})$ & $d 1(\mathrm{~mm})$ & $d 2(\mathrm{~mm})$ & $h(\mathrm{~mm})$ \\
\hline 60 & 15 & 9 & 40 \\
40 & 13.2 & 9.2 & 40 \\
20 & 9.4 & 7.4 & 40 \\
\hline \hline
\end{tabular}

respectively, where the optical anisotropy of quartz is ignored.

\section{EXPERIMENTAL RESULTS}

\section{A. Dependence of radiation intensity on length of medium}

In the present experimental setup two types of radiation are considered. One is Cerenkov radiation and the other is transition radiation from the boundary between the medium and vacuum.

According to the theory of Cerenkov radiation in Sec. II, the intensity is proportional to the length of a medium. On the other hand, since the length of the medium $L$ is larger than the formation length $L_{f}$, the intensity of transition radiation from any kind of boundaries is constant and independent of $L$.

Figure 3 shows the relation of the intensity of the observed radiation and the length of quartz. The circles represents the observed values for the wavelength of $1.3 \mathrm{~mm}$ and the solid line expresses the relation where the intensity is proportional to the length of medium. The observed values are in good agreement with the line and this result confirms that the observed radiation is Cerenkov radiation.

\section{B. Angular distribution of radiation}

The angular distribution of radiation was observed by moving the mirror (M6) in Fig. 2(a). The Cerenkov angle with the $150-\mathrm{MeV}$ electron beam is $62^{\circ}$ for quartz and $44^{\circ}$ for teflon. Then the observed angle corresponding to the Cer-

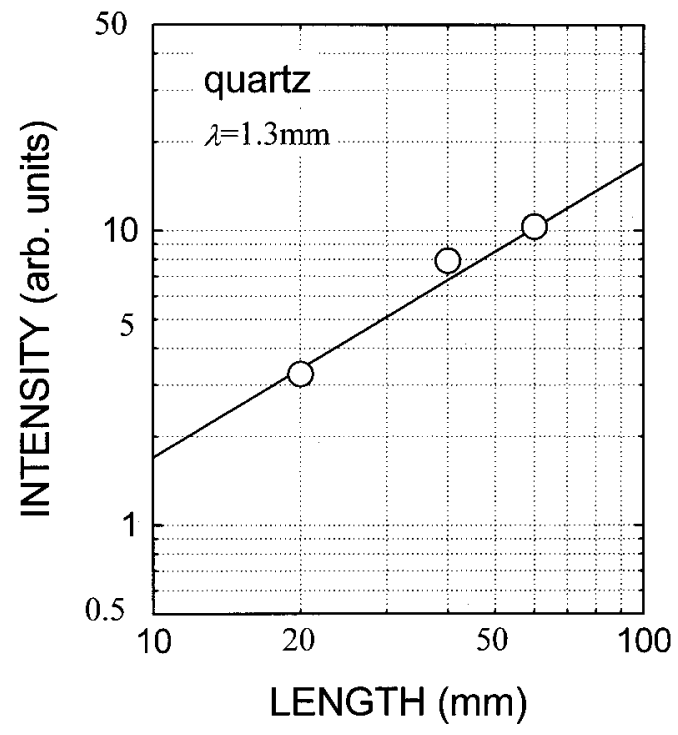

FIG. 3. The relation between the length of quartz and the intensity of radiation. The circles represent the observed data and the solid line expresses linear proportionality of $P$ to $L$.

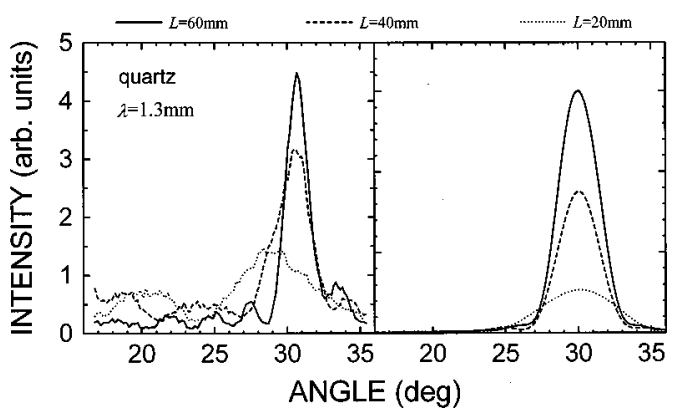

FIG. 4. The dependence of the angular distribution on the length of quartz. The solid, broken, and dotted curves represent the data for quartz of 60,40 , and $20 \mathrm{~mm}$ long, respectively. The curves at the right-hand side show the theoretical calculation.

enkov angle is $30^{\circ}$ and $15^{\circ}$, respectively, for the refraction on the tapered surface of the solid.

The experimental results of the angular distribution of radiation for $\lambda=1.3 \mathrm{~mm}$ for quartz are shown in Fig. 4. The abscissa is the angle from the parallel line of the electron trajectory. The solid, broken, and dotted curves represent the observed data of quartz 60, 40, and $20 \mathrm{~mm}$ long, respectively. The Cerenkov angle $\theta_{c}$ after refraction on the tapered surface of the dielectric is $30^{\circ}$ for the quartz. The corresponding curves at the right hand side in this figure show the theoretical calculation of Cerenkov radiation. The angle at the peak intensity of observed radiation is about $30^{\circ}$ for the quartz 60 and $40 \mathrm{~mm}$ long and is in good agreement with the Cerenkov angle $\theta_{c}$, but it is $29^{\circ}$ for the 20-mm-long quartz. The reason for this small discrepancy was probably due to an inaccurate angle in fixing the quartz to the support.

In order to visualize the satellite peaks of the angular distribution, the ordinate is plotted on a logarithmic scale as shown in Figs. 5(a) for the 60-mm-long quartz and (b) for the 100-mm-long teflon by the solid curves. The intensity has a maximum value at the Cerenkov angle with satellite peaks like a diffraction pattern. The theoretical calculations of Cerenkov radiation are plotted by the broken curves. The satellite peaks are caused by the functional form $(\sin x / x)^{2}$ in Eq. (2.1) of the Cerenkov radiation intensity. The angle at satellite peaks indicated by arrows are in good agreement with the theory. The maximum intensity at the satellite peaks are large in comparison with the theoretical calculation. This may be caused by a stray light.

\section{Dependence of intensity on beam current}

The relation between the intensity and the beam current is shown in Fig. 6. In the present experiment, the current can be expressed by the number $(N)$ of electrons in the bunch, which is also noted in the figure. The circles and the triangles show the observed values for the quartz of $60 \mathrm{~mm}$ long at $\lambda=1.3 \mathrm{~mm}$ and the teflon at $\lambda=2.0 \mathrm{~mm}$, and each intensity was represented on the right and left ordinates, respectively. The solid lines express the quadratic proportionality of $P$ to $N: P \propto N^{2}$.

The observed intensities are proportional to the square of $N$, i.e., to the square of the current. This quadratic dependence confirms that the observed radiation is the coherent radiation expressed by Eq. (2.10). 


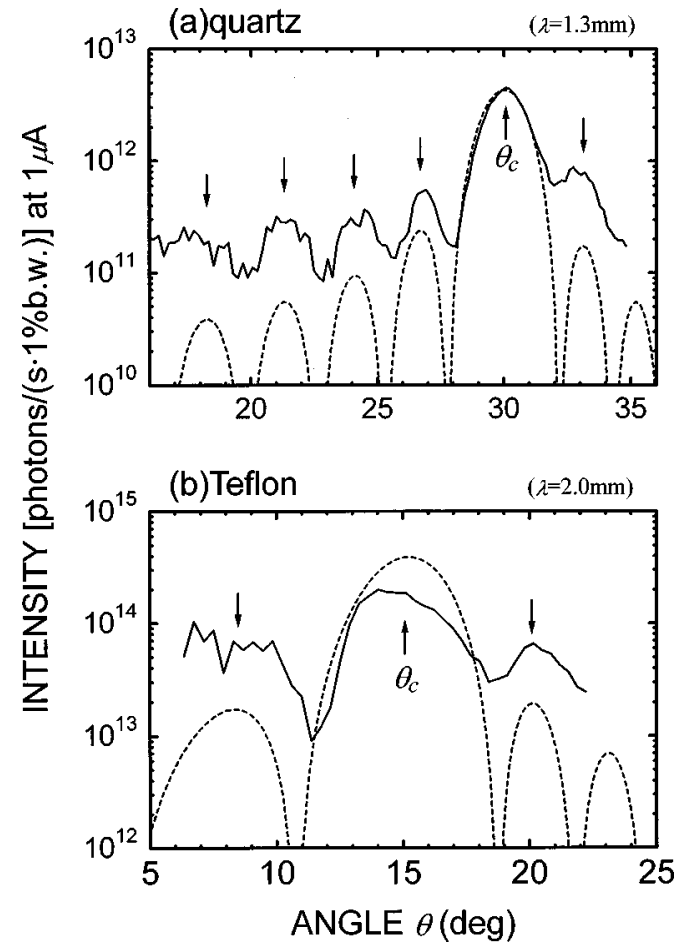

FIG. 5. The angular distribution of radiation from the quartz of $60 \mathrm{~mm}$ long at $\lambda=1.3 \mathrm{~mm}$ and the teflon of $100 \mathrm{~mm}$ long at $\lambda$ $=2.0 \mathrm{~mm}$. The data are plotted on a logarithmic scale in order to visualize satellite peaks in the angular distribution.

\section{Spectrum of radiation}

The spectra of coherent $\breve{C}$ erenkov radiation are shown in Figs. 7(a) and 7(b). The solid curves represent the observed spectra (a) for the quartz $\left(L=60 \mathrm{~mm}, \theta=30^{\circ}\right)$ and (b) for the teflon $\left(L=100 \mathrm{~mm}, \theta=15^{\circ}\right)$, respectively. The intensity of the ordinate shows the number of photons integrated over the acceptance angle of $70 \mathrm{mrad}$, per bandwidth of $1 \%$ (i.e., $\Delta \lambda / \lambda=0.01$ ), and at the average beam current of $1 \mu \mathrm{A}$.

The intensity of Cerenkov radiation from an electron moving parallel to a surface of dielectric was theoretically

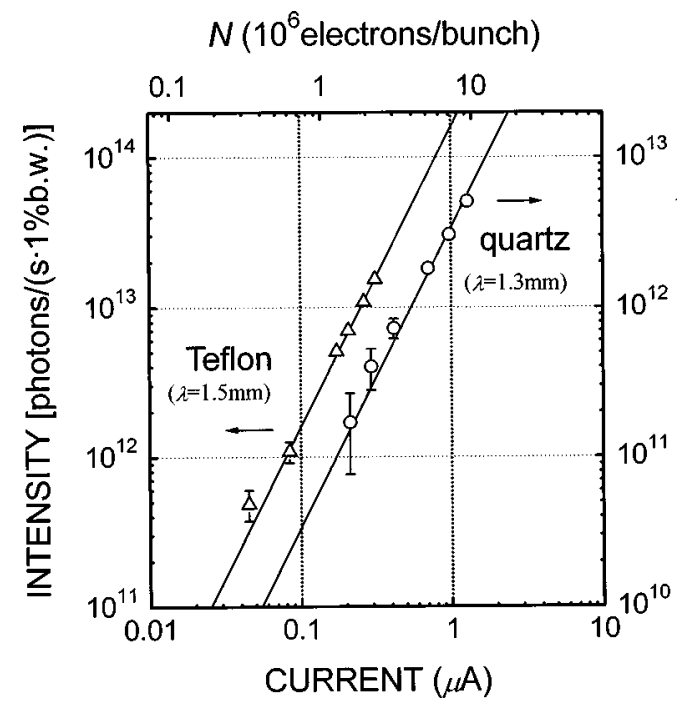

FIG. 6. The relation between the intensity and the beam current. The solid lines represent quadratic dependence of $P$ on $N$.

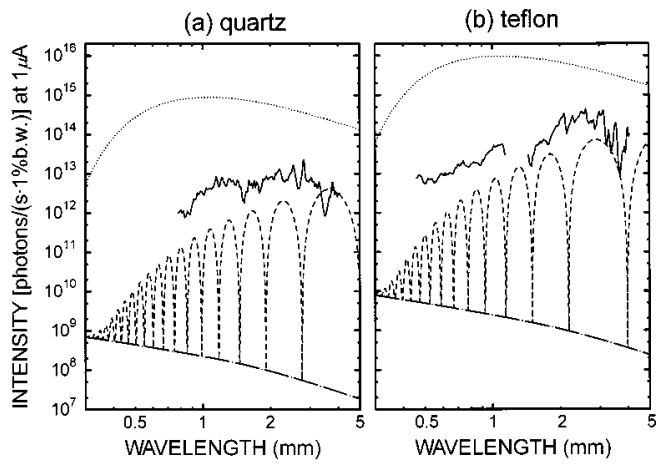

FIG. 7. The spectra of coherent Čerenkov radiation (a) from quartz and (b) from teflon. The solid and dot-dash curves show the observed spectra and the theoretical calculations of incoherent radiation $\left(P=N P_{0}\right)$, respectively. The dotted and broken curves represent the theoretical calculation of coherent Cerenkov radiation on the assumption that the transverse distribution of electrons was negligibly small and was the uniform distribution within the disk of 7 $\mathrm{mm}$ in diameter, respectively. The longitudinal distribution was assumed to be a Gaussian.

derived by Ulrich [26]. Dot-dash-curves in these figures show the theoretical calculation of incoherent radiation, i.e., $P=N P_{0}$. The observed intensities near around $\lambda=3 \mathrm{~mm}$ are enormously enhanced by five orders of magnitude in comparison with the incoherent radiation. However, the enhancement factor is smaller than the number of electrons in a bunch, $7.2 \times 10^{6}$.

For the theoretical calculation of coherent radiation, the longitudinal distribution of electrons in a bunch was assumed to be the Gaussian function as follows:

$$
h(z)=\frac{1}{\sqrt{2 \pi} \sigma_{0}} \exp \left(-\frac{z^{2}}{2 \sigma_{0}^{2}}\right),
$$

where $\sigma_{0}$ is the root-mean-square spread of the Gaussian functions. Since the full width at half maximum of the Gaussian has been determined to be $0.25 \mathrm{~mm}$ from the experiment of coherent synchrotron radiation [3], we used $\sigma_{0}$ $=0.106 \mathrm{~mm}$ in Eq. (4.1). In the cross section of the electron beam, on the other hand, the electrons were assumed to be distributed uniformly within $\rho \leqslant \rho_{0}$ where $\rho_{0}=3.5 \mathrm{~mm}$. Then the longitudinal and transverse bunch form factors are, respectively, given by

$$
f_{L}(\lambda)=\exp \left[-\left(2 \pi \sigma_{0} / \lambda\right)^{2}\right]
$$

and

$$
f_{T}(\lambda)=\left\{\frac{J_{1}\left[2 \pi\left(\rho_{0} / \lambda\right) \sin \theta\right]}{\pi\left(\rho_{0} / \lambda\right) \sin \theta}\right\}^{2},
$$

where $J_{1}$ is the Bessel function of first order. Substituting these form factors into Eq. (2.9), the intensity of coherent Cerenkov radiation is calculated and then the spectra for the quartz and for the teflon are plotted by broken curves in Figs. 7 (a) and 7(b). The divergence factor $\chi$ in Eq. (2.9) was approximated to unity because the angular divergence of the 
electron beam was negligible. The calculated curves oscillate with wavelength, but the envelope of the oscillation are roughly similar to that of the observed spectra.

On the other hand, the dotted curves represent the theoretical calculation when the cross-sectional size of the electron beam is negligibly small, i.e., $f_{T}=1$. The assumption was valid on the spectrum of the coherent synchrotron radiation [3] and transition radiation [9] with the electron beam of the Tohoku Linac. However, since the Cerenkov radiation from the solid dielectrics is emitted with the large Cerenkov angle $\theta$, the electron distribution in the cross section plays a significant role in the investigation of the coherence effect. The discrepancy of intensity between the observed spectrum and the theoretical one is due to the inaccuracy of the trans- verse distribution of electrons which was assumed in the theoretical calculation.

\section{ACKNOWLEDGMENTS}

We express our gratitude to Dr. T. Nakazato and Professor T. Yamakawa of the Laboratory of Nuclear Science, Tohoku University, for useful discussions. We also thank T. Tsutaya of the Research Institute for Scientific Measurements, Tohoku University, and S. Urasawa, A. Kurihara, S. Takahashi, and Y. Shibasaki of the Laboratory of Nuclear Science, Tohoku University, for their technical support and assistance. This work was partially supported by a Grant-inAid for Scientific Research from the Ministry of Education, Science and Culture of Japan.
[1] T. Nakazato, M. Oyamada, N. Niimura, S. Urasawa, O. Konno, A. Kagaya, R. Kato, T. Kamiyama, Y. Torizuka, T. Nanba, Y. Kondo, Y. Shibata, K. Ishi, T. Ohsaka, and M. Ikezawa, Phys. Rev. Lett. 63, 1245 (1989).

[2] Y. Shibata, K. Ishi, T. Ohsaka, H. Mishiro, T. Takahashi, M. Ikezawa, Y. Kondo, T. Nakazato, M. Oyamada, N. Niimura, S. Urasawa, R. Kato, and Y. Torizuka, Nucl. Instrum. Methods Phys. Res. A 301, 161 (1991).

[3] K. Ishi, Y. Shibata, T. Takahashi, H. Mishiro, T. Ohsaka, M. Ikezawa, Y. Kondo, T. Nakazato, S. Urasawa, N. Niimura, R. Kato, Y. Shibasaki, and M. Oyamada, Phys. Rev. A 43, 5597 (1991).

[4] Y. Shibata, T. Takahashi, K. Ishi, F. Arai, H. Mishiro, T. Ohsaka, M. Ikezawa, Y. Kondo, S. Urasawa, T. Nakazato, R. Kato, S. Niwano, and M. Oyamada, Phys. Rev. A 44, R3445 (1991).

[5] E.B. Blum, U. Happek, and A.J. Sievers, Nucl. Instrum. Methods Phys. Res. A 307, 568 (1991).

[6] U. Happek, A.J. Sievers, and E.B. Blum, Phys. Rev. Lett. 67, 2962 (1991).

[7] Y. Shibata, K. Ishi, T. Takahashi, T. Kanai, M. Ikezawa, K. Takami, T. Matsuyama, K. Kobayashi, and Y. Fujita, Phys. Rev. A 45, R8340 (1992).

[8] T. Takahashi, Y. Shibata, F. Arai, K. Ishi, T. Ohsaka, M. Ikezawa, Y. Kondo, T. Nakazato, S. Urasawa, R. Kato, S. Niwano, and M. Oyamada, Phys. Rev. E 48, 4674 (1993).

[9] Y. Shibata, K. Ishi, T. Takahashi, T. Kanai, F. Arai, S. Kimura, T. Ohsaka, M. Ikezawa, Y. Kondo, R. Kato, S. Urasawa, T. Nakazato, S. Niwano, M. Yoshioka, and M. Oyamada, Phys. Rev. E 49, 785 (1994).

[10] Y. Shibata, T. Takahashi, T. Kanai, K. Ishi, M. Ikezawa, J. Ohkuma, S. Okuda, and T. Okada, Phys. Rev. E 50, 1479 (1994).

[11] Y. Shibata, S. Hasebe, K. Ishi, T. Takahashi, T. Ohsaka, M. Ikezawa, T. Nakazato, M. Oyamada, S. Urasawa, T. Yamakawa, and Y. Kondo, Phys. Rev. E 52, 6787 (1995).

[12] K. Ishi, Y. Shibata, T. Takahashi, S. Hasebe, M. Ikezawa, K. Takami, T. Matsuyama, K. Kobayashi, and Y. Fujita, Phys. Rev. E 51, R5212 (1995).
[13] Y. Shibata, S. Hasebe, K. Ishi, S. Ono, M. Ikezawa, T. Nakazato, M. Oyamada, S. Urasawa, T. Takahashi, T. Matsuyama, K. Kobayashi, and Y. Fujita, Phys. Rev. E 57, 1061 (1998).

[14] T. Takahashi, T. Matsuyama, K. Kobayashi, Y. Fujita, Y. Shibata, K. Ishi, and M. Ikezawa, Rev. Sci. Instrum. 69, 3770 (1998).

[15] Y. Shibata, K. Ishi, S. Ono, Y. Inoue, S. Sasaki, M. Ikezawa, T. Takahashi, T. Matsuyama, K. Kobayashi, and Y. Fujita, Phys. Rev. Lett. 78, 2740 (1997).

[16] Y. Shibata, K. Ishi, S. Ono, Y. Inoue, S. Sasaki, M. Ikezawa, T. Takahashi, T. Matsuyama, K. Kobayashi, Y. Fujita, and E.G. Bessonov, Nucl. Instrum. Methods Phys. Res. B 145, 49 (1998).

[17] I.E. Tamm and I.M. Frank, Dokl. Akad. Nauk SSSR 14, 107 (1937).

[18] J.R. Neighbours, F.R. Buskirk, and A. Saglam, Phys. Rev. A 29, 3246 (1984).

[19] J. Ohkuma, S. Okuda, and K. Tsumori, Phys. Rev. Lett. 66, 1967 (1991).

[20] Y. Shibata, K. Ishi, T. Takahashi, F. Arai, M. Ikezawa, K. Takami, T. Matsuyama, K. Kobayashi, and Y. Fujita, Phys. Rev. A 44, R3449 (1991).

[21] T. Takahashi, T. Kanai, Y. Shibata, K. Ishi, M. Ikezawa, T. Nakazato, M. Oyamada, S. Urasawa, T. Yamakawa, K. Takami, T. Matsuyama, K. Kobayashi, and Y. Fujita, Phys. Rev. E 50, 4041 (1994).

[22] P.D. Coleman and C. Enderby, J. Appl. Phys. 31, 1695 (1960).

[23] J.E. Walsh, T.C. Marshall, and S.P. Schlesinger, Phys. Fluids 20, 709 (1997)

[24] I.E. Tamm, J. Phys. (Moscow) 1, 439 (1939).

[25] V.P. Zrelov, M. Klimanova, V.P. Lupiltsev, and J. Ružička, Nucl. Instrum. Methods 215, 141 (1983).

[26] R. Ulrich, Z. Phys. 194, 180 (1966).

[27] L.A. Vardanyan, G.M. Garibyan, and C. Yang, Izv. Akad. Nauk Arm. SSR, Fiz. 10, 350 (1975).

[28] Y. Shibata, T. Takahashi, T. Kanai, K. Ishi, M. Ikezawa, J. Ohkuma, S. Okuda, and T. Okada, Phys. Rev. E 50, 1479 (1994). 